\title{
JAX, M.D. A Framework for Differentiable Physics
}

\author{
Samuel S. Schoenholz \\ Google Research: Brain Team \\ schsam@google.com
}

\author{
Ekin D. Cubuk \\ Google Research: Brain Team \\ cubuk@google.com
}

\begin{abstract}
We introduce JAX MD, a software package for performing differentiable physics simulations with a focus on molecular dynamics. JAX MD includes a number of physics simulation environments, as well as interaction potentials and neural networks that can be integrated into these environments without writing any additional code. Since the simulations themselves are differentiable functions, entire trajectories can be differentiated to perform meta-optimization. These features are built on primitive operations, such as spatial partitioning, that allow simulations to scale to hundreds-of-thousands of particles on a single GPU. These primitives are flexible enough that they can be used to scale up workloads outside of molecular dynamics. We present several examples that highlight the features of JAX MD including: integration of graph neural networks into traditional simulations, metaoptimization through minimization of particle packings, and a multi-agent flocking simulation. JAX MD is available at www.github.com/google/jax-md.
\end{abstract}

\section{Introduction}

The past few years have seen an explosion of progress at the intersection of machine learning, automatic differentiation, and the physical sciences [1,2]. Deep neural network models of quantum mechanical energies have become ever more accurate [3-11], graph networks have been designed to simulate systems by observing their dynamics [12-15], and physics-inspired models have clarified the connection between structure and dynamics in complex systems such as glasses [16-19] and proteins [20,21]. Combining classical simulation environments with deep learning or optimizing them directly via automatic differentiation has led to significant advances including: end-to-end learning of protein structures [22, 23], the inverse design of photonic crystals [24], and structural optimization via reparameterization using convolutional networks [25]. Finally, significant progress in developmental psychology has centered around combining intuitive physics simulations with probabilistic programming [26].

This flurry of excellent research has highlighted a significant source of inefficiency: we lack general purpose simulation environments that can easily be integrated with existing machine learning tools. For example, despite the rapid progress developing neural network models of quantum mechanical systems, it is difficult to incorporate these models into classical simulation environments such as LAMMPS [27], HOOMD-Blue [28, 29], and OpenMM [30]. Currently, integrating machine learning models into existing simulations requires the construction of custom software "bridges" [31-36]. This is a hindrance to the adoption of machine learning methods in practice. Moreover, most research into differentiable simulation environments are bespoke pieces of software written for a single application. As such, currently research at the intersection of simulation and machine learning requires practitioners to be experts, not only at machine learning, but also at writing the simulations themselves. 
Here we introduce JAX MD, which is a software package that makes it easy to write physics simulations that naturally integrate with machine learning models in the flourishing JAX [37, 38] ecosystem. At its core, JAX MD provides a collection of lightweight primitive operations that are useful in developing and analyzing a broad range of physics simulations. Building on these primitives, JAX MD provides standard molecular dynamics simulation environments and interaction potentials. To help researchers analyze simulations, we include tools that can be used visualize particle trajectories within Jupyter notebooks [39]. Finally, we provide several widely-used neural network architectures that can be incorporated into simulations no modifications of the simulation code.

We begin with a brief overview of JAX and JAX MD. We then provide benchmarks against classic simulation software before describing the structure of the library. Finally, we discuss several examples that illustrate the use of JAX MD to perform research at the intersection of simulation and machine learning including:

- Training and deployment of a neural network potential in simulation.

- Differentiation through simulation for structural optimization.

- A simulation of flocking behavior.

Each example features an accompanying Colaboratory notebook that reproduces the results and includes more details about the use-case. While these examples are designed to be illustrative, they involve issues faced by researchers in practice. Moreover, each of these examples would be difficult to implement using existing simulation infrastructure.

\section{Related Work}

Automatic differentiation has enjoyed a rich history in the physical sciences [40]; recent applications include: structural optimization [25], quantum chemistry [41], fluid dynamics [42-44], computational finance [45], atmospheric modelling [46, 47], optimal control [48], physics engines [49], protein modelling [22, 23], and quantum circuits [50]. Tools such as DeepChem [51] have consolidated useful best practices for applying deep learning to chemistry. Despite significant work on the topic and a large number of high quality automatic differentiation libraries [52-56, 38, 57], the number of general purpose simulation environments that are integrated with automatic differentiation is scarce.

One exciting recent development in this direction in DiffTaichi [58]. DiffTaichi augments the newly developed Taichi [59] programming language with automatic differentiation capabilities. The Taichi language is a performance oriented language for writing simulations and additional automatic differentiation capabilities offer promising opportunities. There are several contrasts between JAX MD and DiffTaichi. First, JAX MD aims to provide simulation capabilities to a strong machine learning library. Thus, we expect to have easier integration with machine learning models and more flexible automatic differentiation support. Second, JAX MD provides simulation environments that are ready-to-use for research in the physical sciences. However, since Taichi is built from the ground up to deliver fast simulations we expect DiffTaichi to offer a performance advantage relative to JAX MD for many workloads. Nonetheless, as we discuss below, the design of JAX and XLA allows us to produce high-performance code that is competitive with existing software.

\section{Architecture}

We begin by briefly describing JAX before discussing the architectural choices we made in designing JAX MD. JAX is the successor to Autograd and shares key design features. As with Autograd, the main user-facing API of JAX is in one-to-one correspondence with Numpy [60], the ubiquitous numerical computing library for Python. On top of this, JAX includes a number of higher-order function transformations that can be applied to any function written in terms of JAX primitives. Examples of such transformations are: automatic differentiation (grad), vectorization on a single device (vmap), parallelization across multiple devices (pmap), and just-in-time compilation ( $j i t)$. These function transformations are arbitrarily composable, so one can write e.g. jit $(\operatorname{vmap}(\operatorname{grad}(f)))$ to just-in-time compile a function that computes per-example gradients of a function $f$. JAX makes heavy use of the accelerated linear algebra library, XLA, which allows compiled functions to be run with a single call on CPU, GPU, or TPU. This effectively removes execution speed issues that 
generally face Python programs. Moreover, this just-in-time compilation allows XLA to perform whole-program optimizations that can considerably improve execution speed.

Unlike most physics simulations, JAX MD adopts a functional style similar to JAX with immutable data and first-class functions. In a further departure from most physics software, JAX MD features no classes or object hierarchies and instead uses a data driven approach that involves transforming arrays of data. JAX MD often uses dataclasses to provide minimal organization to collections of arrays. This functional and data-oriented approach complements JAX and makes it easy to apply the range of function transformations that JAX provides. JAX MD makes extensive use of automatic differentiation and automatic vectorization to concisely express ideas (e.g. force as the negative gradient of energy) that are challenging in more conventional libraries. Additionally, we include a number of higher-order functions that make it particularly easy to define custom interaction potentials. Since JAX MD leverages XLA to compile whole simulations to single device calls, it can be entirely written in Python while still being fast enough to perform research. Together this means that simulations implemented in JAX MD tend to be very concise and look like textbook descriptions of the subject.

\subsection{Benchmarks}

While our use of JAX and XLA may provide a more productive research environment than conventional simulation packages, it does have several drawbacks. Most significantly, the primitives exposed by XLA are sometimes at odds with computations that are commonplace in molecular dynamics. Notably, XLA requires that shapes be statically known at compile-time and it is often challenging to use complex data-structures. While JAX MD is fast enough for many research applications, since XLA has been optimized for machine learning workloads, JAX MD is still slower than production quality MD packages using custom CUDA kernels. This is especially true on the CPU backend, where JAX MD currently struggles to scale to large systems. Here we include benchmarks against two of the most common and high-performance molecular dynamics simulation environments: LAMMPS and HOOMD-Blue.

To compare performance, we simulate Lennard-Jones particles using JAX MD, LAMMPS, and HOOMD-Blue. This is a ubiquitous performance benchmark in the molecular dynamics literature. In each case we consider a system of $N$ particles simulated for 10,000 steps. We include a small $N=216$ particle system as well as a larger system on each platform. For the larger simulations we used neighbor lists with a cutoff of $3 \sigma$ and a halo of $0.5 \sigma$. LAMMPS updates the neighbor list every 10 steps whereas both HOOMD-Blue and JAX MD update their neighbor list whenever a particle moves by more than the halo distance.

\begin{tabular}{|l|r|r|r|r|r|r|}
\hline & \multicolumn{2}{|c|}{ CPU } & \multicolumn{2}{c|}{ K80 GPU } & \multicolumn{2}{c|}{ TPU } \\
\cline { 2 - 7 } & $\mathrm{N}=216$ & $\mathrm{~N}=10 \mathrm{k}$ & $\mathrm{N}=216$ & $\mathrm{~N}=64 \mathrm{k}$ & $\mathrm{N}=216$ & $\mathrm{~N}=10 \mathrm{k}$ \\
\hline JAX MD & 1.9 & 700 & 0.09 & 10.5 & 0.02 & 360 \\
LAMMPS & 0.1 & 120 & 0.20 & 4.0 & - & - \\
HOOMD-BLUE & 0.2 & 120 & 0.07 & 4.5 & - & - \\
\hline
\end{tabular}

Table 1: Comparison of performance between JAX MD, HOOMD Blue, and LAMMPS. N denotes the number of atoms used in the simulation. Time is reported in units of milliseconds-perstep.

We list the various performance benchmarks in table 1. For small systems on GPU we see that JAX MD performs comparably to the two classic simulation environments. On larger systems, JAX MD incurs approximately a $2.4 \times$ slowdown. On CPU, we see that JAX MD is about a factor of 10 slower than either HOOMD-Blue or LAMMPS. On TPU we see that JAX MD performs well for small systems (although a quantitative comparison is difficult, since TPU has limited support for 64-bit arithmetic). However, TPUs perform badly on large systems due to poor neighbor list performance. Future work will focus on improving execution speed on CPU and TPU.

\subsection{Primitives}

We now discuss the structure of JAX MD, starting with primitive operations that are generally useful for constructing simulations based on elements interacting in two- or three-dimensions. This is not 
meant to be an exhaustive discussion of the features of JAX MD, but is meant to highlight particularly salient points.

\subsubsection{Spaces}

To describe a physical system we must first define the space in which the system lives. In many cases, this space is constructed from subsets of $\mathbb{R}^{D}$ with $D=2$ or $D=3$. For notational convenience and concreteness we will focus on the two-dimensional setting, but we note that extensions to higher dimensions are trivial. In the simplest setting, the space will simply be all of $\mathbb{R}^{D}$ and these are called "free" boundary conditions. In many cases it is useful to incorporate "periodic boundary conditions". When using periodic boundary conditions, the space is a rectangle $U=\left[L_{x}, 0\right] \times\left[0, L_{y}\right]$ with the association that for any vector $\vec{r}_{i} \in U$ and $n_{x}, n_{y} \in \mathbb{Z}, \vec{r}_{i}=\vec{r}_{i}+n_{x} L_{x} \vec{e}_{x}+n_{y} L_{y} \vec{e}_{y} . U$ together with this identity forms periodic boundary conditions in which elements can "wrap around" the sides of the space. Topologically, this describes a space that is homeomorphic to a two-torus.

In JAX MD we describe spaces by a pair of functions. First, a function $\vec{d}: \mathbb{R}^{D} \times \mathbb{R}^{D} \rightarrow \mathbb{R}^{D}$ computes the displacement between two particles. This function can in turn be used to define a metric on the space $d: \mathbb{R}^{D} \times \mathbb{R}^{D} \rightarrow \mathbb{R}$ by $d(\vec{a}, \vec{b})=\|\vec{d}(\vec{a}, \vec{b})\|_{2}$. Note, that in systems with periodic boundary conditions $\vec{d}$ computes the displacement between a particle and the nearest "image" of the second particle. Second, a shift function $\mu: \mathbb{R}^{D} \times \mathbb{R}^{D} \rightarrow \mathbb{R}^{D}$ must be defined that moves a particle by a displacement vector. In JAX MD the boundary conditions discussed above are implemented as $\mathrm{d}, \mathrm{mu}=$ space.free () and $\mathrm{d}, \mathrm{mu}=$ space $\cdot$ periodic $(\mathrm{L})$ respectively.

The displacement functions are defined for a single pair of particles. However, it is often desirable to compute the displacement for all pairs in a set of $N$ particles, $d_{\text {pair }}: \mathbb{R}^{N \times D} \times \mathbb{R}^{N \times D} \rightarrow \mathbb{R}^{N \times N \times D}$, between a set of particles and their neighbors, $d_{\text {nbrs }}: \mathbb{R}^{N \times D} \times \mathbb{R}^{N \times N_{\text {nbrs }} \times D} \rightarrow \mathbb{R}^{N \times N_{\text {nbrs }} \times D}$, or between ordered pairs $d_{\text {bond }}: \mathbb{R}^{N \times D} \times \mathbb{R}^{N \times D} \rightarrow \mathbb{R}^{N \times D}$. For convenience JAX MD provides several light-weight wrappers around JAX's automatic vectorization that allow us to transform displacement functions for a pair of particles into these respective forms: d_pair = space.map_pair(d), d_nbrs = space $\cdot$ map_neighbors $(d)$, and d_bond $=$ space $\cdot$ map_bond $(d)$.

\subsubsection{Interactions ${ }^{1}$}

A key component of any simulation is the definition of interactions between elements of the simulation. The most common way of defining interactions in physics is via an energy function (similar to a loss for a neural network). Given a collection of $N$ elements, $\vec{r}_{i} \in \mathbb{R}^{D}$ with $1 \leq i \leq N$, an energy is a function $U: \mathbb{R}^{N \times D} \rightarrow \mathbb{R}$. With the energy in hand one can then perform a wide range of simulations using variants on Newton's laws: $m \ddot{\overrightarrow{r_{i}}}=-\nabla_{\vec{r}_{i}} U$ where $m$ is the mass. Rather than defining an energy as an interaction between all the elements of the simulation simultaneously, it is common to define a "pairwise" energy based on the displacement between a pair of elements, $u\left(\vec{r}_{i}-\vec{r}_{j}\right)$. In this case the total energy is then defined by the sum over pairwise interactions, $U=\frac{1}{2} \sum_{i \neq j} u\left(\vec{r}_{i}-\vec{r}_{j}\right)$.

In JAX MD the energy can be any differentiable function and forces are computed using automatic differentiation. To facilitate the construction of energy functions from pairwise interactions, JAX MD provides several higher-order functions that take functions that act on pairs of elements and transforms them to operate on an entire system. In analogy to the vectorization of spaces, JAX MD provides the functions $U=\operatorname{smap} \cdot \operatorname{pair}(d, u), U=\operatorname{smap} \cdot \operatorname{neighbors}(d, u)$, and $U=\operatorname{smap} \cdot \operatorname{bond}(d, u)$ that take a pairwise function and promotes it to act on all pairs, all neighbors, or ordered pairs respectively.

\subsubsection{Spatial Partitioning}

In the analysis and simulation of physical systems, many quantities of interest are local in the sense that they depend only on pairs of elements, $(i, j)$, such that $\left\|\vec{r}_{i}-\vec{r}_{j}\right\|<\sigma$. In this case, it is wasteful to compute the result for every pair of elements, which scales as $\mathcal{O}\left(N^{2}\right)$, since the number of nonzero contributions often only scales as $O(N)$. To facilitate these computations, JAX MD includes two standard primitive operations to spatially partition systems into neighborhoods of size $\sigma$ : first, partition.cell_list takes a collection of elements and places them into "cells" where each cell is has size $\sigma$; second, partition.neighbor_list converts lists of positions into lists of neighboring

\footnotetext{
${ }^{1}$ Colaboratory notebook at github.com/google/jax-md/notebooks/custom_potentials.ipynb
} 
elements within $\sigma$. Note that partition.neighbor_list uses partition.cell_list under the hood to construct the list of neighbors in $\mathcal{O}(N \log N)$ time.

\subsubsection{Neural Networks}

We include several neural network components, built on top of Haiku [61], that make it easy to build neural networks that can be incorporated into simulations. In particular, we provide code to compute popular featurizations that have been used in the literature [62, 63, 16, 64]. Using these features we provide an implementation of the Behler-Perrinello architecture [65], which is a popular neural network for approximating quantum mechanical energies. We also provide a number of building blocks to construct graph neural networks based on the GraphNet library [66] along a standard graph network recently used to model glasses [19].

\subsection{Higher Level Functions}

We now briefly describe functions that can be used to perform molecular physics simulations. Here the elements of the simulation will typically be particles or atoms.

\subsubsection{Dynamics and Simulations}

JAX MD supports simple constant energy (NVE) simulation as well as Nose-Hoover [67], Langevin, and Brownian simulations at constant temperature (NVT). JAX MD also supports Fast Inertial Relaxation Engine (Fire) Descent [68] and Gradient Descent to minimize the energy of systems. All simulations in JAX MD follow a pattern that is inspired by JAX's optimizers. In particular, simulations are defined by pairs of two functions: an initialization function and an update function. For example, to construct an NVE simulation one would write, init_fn, update_fn = simulate.nve (energy_fn, shift_fn, $d t=1 e-3)$. The initialization function, state = init_fn(key, positions), takes particle positions and returns an initial simulation state; the update function, state = update fnn $_{\text {(state }}$ ), takes a state and applies a single update step to the state. Keyword arguments fed to the update function get passed to energy functions as well as the displacement and shift functions. This is useful, for example, to simulate a system with a time-varying potential by writing state $=$ update $\_$fn $($state, $\mathrm{t}=\mathrm{t})$.

\subsubsection{Energy Functions}

We provide a number of energy functions including several pair potentials - Lennard-Jones [69], soft-sphere [70], Buckingham-type [71-73], Gupta [74], and Morse [75] - as well as the StillingerWeber [76] three-body potential, the Embedded Atom [77] many-body potential, and soft-spring bonds. For pairwise potentials, we provide three functions: one giving the functional form (e.g. $\mathrm{E}=$ soft_sphere (dr)), one thin wrapper around smap. pair that maps the form to all pairs of particles (e.g. energy_fn = soft_sphere_pair (d) where $d$ is a displacement function), and one thin wrapper around smap.neighbors that maps the form over all neighbors (e.g. neighbor_fn, energy_fn $=$ soft_sphere_neighbor_list(d, box_size)). We also include wrappers around the neural network components to build neural networks specifically designed to predict energies as described in section 4.1. Forces can easily be computed using a helper function quantity.force (energy_fn) which is a thin wrapper around jax.grad. The automatic computation of derivatives and vectorization make it significantly easier to add new potentials to JAX MD than it is to add potentials to classical MD packages.

\section{Three Vignettes}

\subsection{Neural Network Potentials ${ }^{2}$}

In this section we describe the training and deployment of two state-of-the-art neural networks: the Behler-Parrinello neural network [65] and a graph neural network. We will consider a common task in the field of empirical potentials research: fitting a neural network model to quantum mechanical energies and forces. We will use open source data from a recent study of silicon in different crystalline

\footnotetext{
${ }^{2}$ Colaboratory notebook at: github.com/google/jax-md/notebooks/neural_networks.ipynb
} 
phases [78]. Here, Density Functional Theory (DFT) - the standard first principles quantum mechanics technique - was used to simulate 64 Silicon atoms at three different temperatures. We follow a standard procedure in the field and uniformly sample these trajectories to create 50k configurations that we split between a training set, a validation set, and a test set.

To create our neural network potential we can write init_fn, energy_fn = energy.behler_parrinello(d) or init_fn, energy_fn = energy.graph_network(d) which uses Haiku [61] to construct the energy model. The model itself is given by two functions: the initialization function, params = init_fn(key, input_shape), instantiates parameters of the model; the energy function, $E=$ energy_fn (params, $R$ ), evaluates the model given parameters and positions. Here the energy function evaluates the energy for a single configuration; however, for training we often want to evaluate the model over an entire batch of data. To do this, we can use JAX's automatic vectorization as vectorized_energy_fn $=\operatorname{vmap}($ energy_fn, $($ None, 0$)$ ) to vectorize only over the positions.

For the Behler-Parrinello architecture we train for 800 epochs using momentum with learning rate $5 \times 10^{-5}$ and batch size 10. For the GNN we train for 160 epochs using ADAM with a learning rate of $1 \times 10^{-3}$ and batch size 128. We use a loss that is the sum of two terms: the Mean Squared Error (MSE) between the network outputs and the quantum mechanical energies and the MSE between the forces predicted by the model and the quantum mechanical forces. While the Behler-Parrinello network is explicitly invariant to global rotations, the graph neural network is not. Therefore, for the graph neural network we additionally augment the data with random rotations and flips.
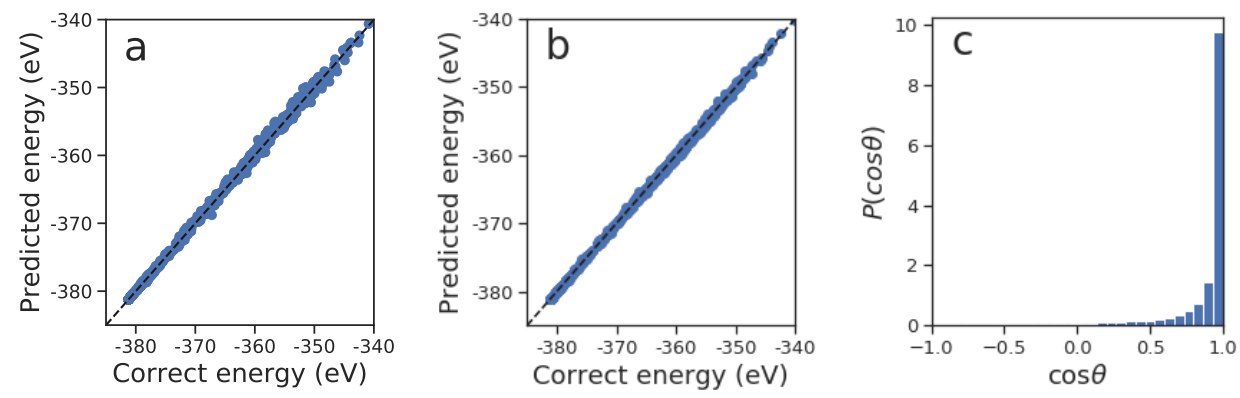

Figure 1: Empirical potentials trained on DFT. (a) shows the agreement between predicted energies by a Behler-Parrinello neural network and the correct DFT energies for test samples. (b) shows the agreement between energies predicted by the GNN and the correct DFT energies. (c) the distribution of the per-particle cosine-angle between the correct force and the predicted force for the graph neural network.

We plot the result of training these two networks in fig. 4.1. Fig. 4.1 (a) and (b) show the predicted energy on the test set against the ground truth labels for both network architectures. We find that the graph neural network achieves a root-mean-squared error of $2.8 \mathrm{meV} /$ atom. We can construct forces for these models using JAX MD along with JAX's automatic differentiation as force_fn = quantity.force (energy_fn). We emphasize that the step of computing forces would be extremely laborious in traditional MD packages. In fig. 4.1 (c) we show the cosine-angle between the forces computed from the graph neural network model and the ground-truth forces computed using density functional theory. We observe strong correlations between the predicted forces and the exact forces.

Now that the model is trained, we can easily deploy it into our simulations. To do this, it is useful to "bake in" the parameters using Python's partial evaluation, energy_fn = functools.partial (energy_fn, params). This energy function can now be used as the input to any of the JAX MD simulation environments. To demonstrate this, we simulate a larger system of 64,000 atoms using the graph neural network to compute forces. The graph neural network provides accurate energies and forces for a system that is far larger than could possibly be simulated with DFT. A snapshot of this simulation is shown in fig. 2. 


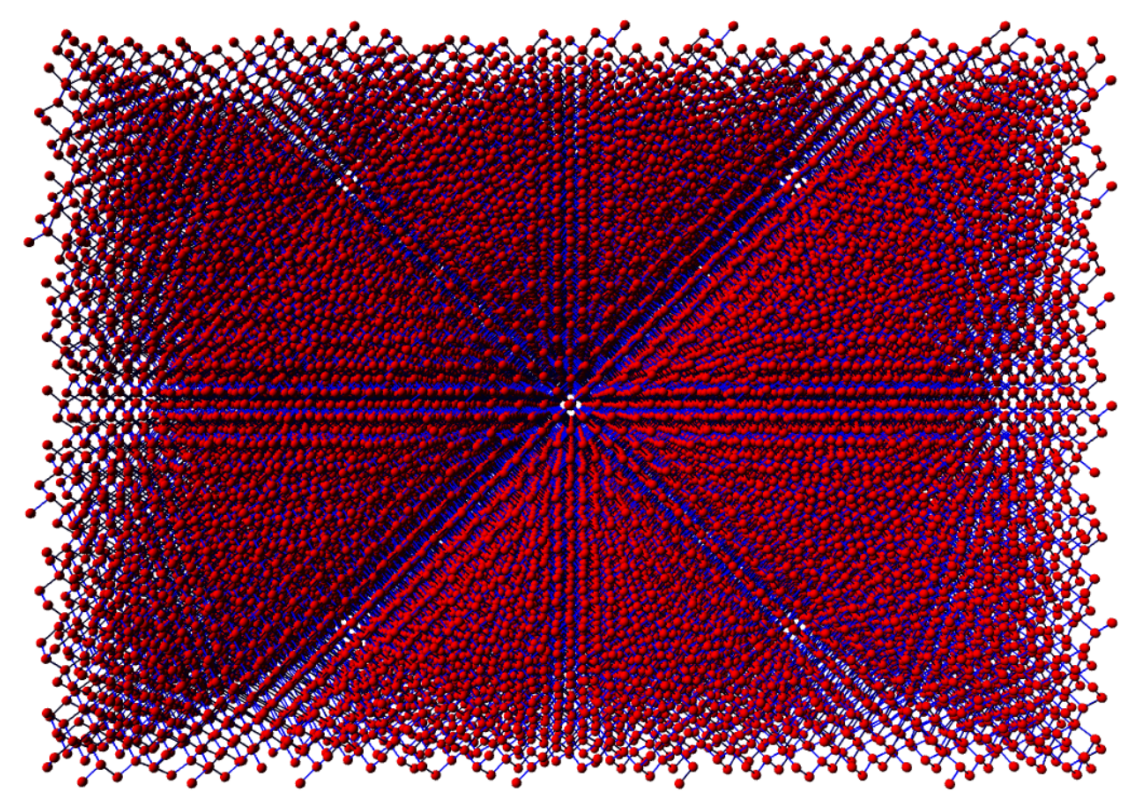

Figure 2: Simulation using a GNN. Here we show a snapshot of a 64,000 atom NVT simulation using a graph neural network energy function trained on DFT data.

\subsection{Optimization Through Dynamics ${ }^{3}$}

Here we describe how one can differentiate through a simple simulation to optimize the parameters of the simulation. We will use a simple model that is widely studied in condensed matter physics: the packing of spheres into a box. In two-dimensions, when soft disks of equal radius are compressed inside a box they pack regularly into a hexagonal grid. When the spheres have different sizes, however, the hexagonal order can be interrupted and the resulting structure becomes random. These random structures are often referred to as "jammed" [70]. We will consider a system with spheres of two distinct sizes: half of the disks will have diameter 1 and half will have diameter $D$. We will adjust the size of the simulation box so that the fraction of space taken up by the disks remains invariant as we tune $D$.

In fig. 3 (a) we see energy minimizing configurations for several different values of $D$. When $D=1$ (bottom right) the system has its characteristic hexagonal order and as $D$ shrinks the configuration gets more disordered until it reaches a maximally frustrated state (top right). As the small particles are further shrunk the system becomes more ordered with local hexagonal order among the large particles and the small particles fitting into interstices (top left). Fig. 3 (b) shows the energy of local minimia, averaged over 100 different random samples, as a function of $D$. We see that the energy achieves a maximum where the system is maximally frustrated. We would like to find this point of maximum frustration by differentiating through the simulation directly.

To do this we write a function final_energy $=$ minimize_energy (diameter, key) that takes a diameter for the small particles as well as a random key (the code for this function is provided in the section A). The function generates a random configuration of particles, minimizes them to their nearest minimum, and then returns the energy of the final configuration. To generate fig. 3 (b) the minimization function can be vectorized separately over diameters and random keys,

vectorized_minimize_energy $=\operatorname{vmap}\left(\operatorname{vmap}\left(\operatorname{minimize} \_\right.\right.$energy, $($None, 0$\left.)\right),(0$, None $\left.)\right)$

Since each energy calculation is the result of a differentiable simulation, we can differentiate through the minimization with respect to $D$. This allows us to find extrema of the minimized-energy as a function of diameter using first-order optimization methods. This is implemented in JAX MD

${ }^{3}$ Colaboratory notebook at: github.com/google/jax-md/notebooks/meta_optimization.ipynb 

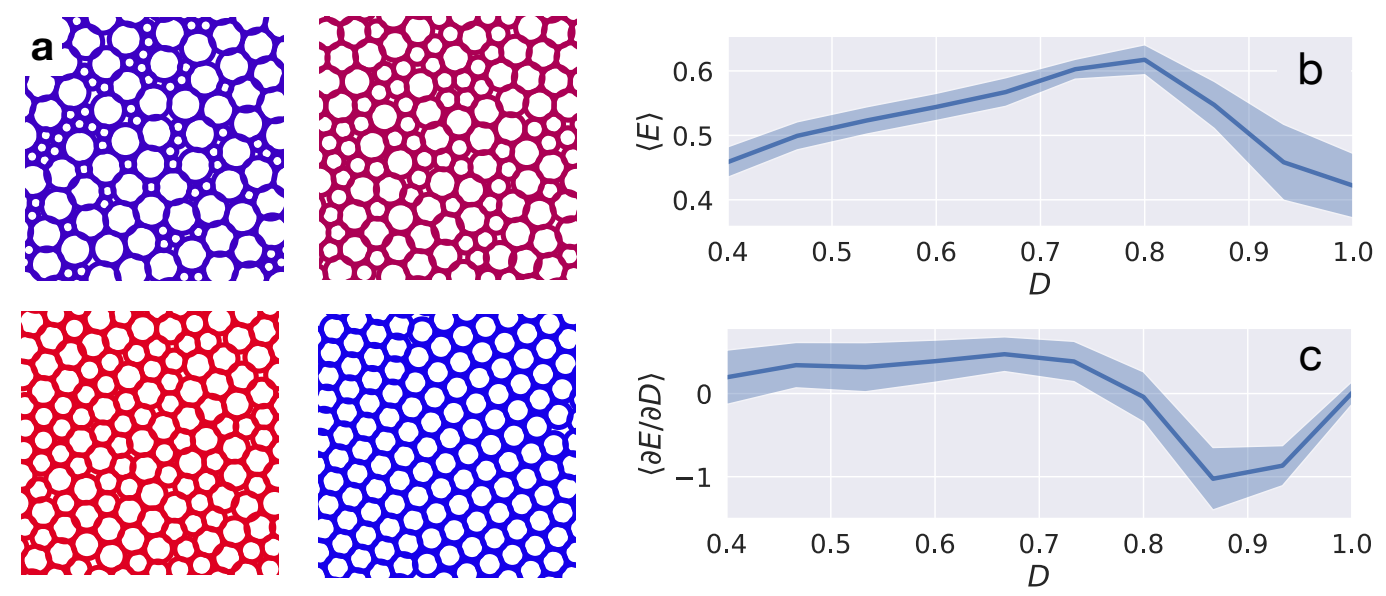

Figure 3: Finding maximally frustrated states. (a) Configurations for varying small-particle diameter $D$. (b) The average energy and the standard deviation of the energy at $D$. (c) The derivative we calculate by differentiating through energy optimization by gradient descent as a function of $D$.

as, $d E_{-} d D_{-} f n=\operatorname{grad}\left(\operatorname{minimize} \_\right.$energy). Of course the $d E_{-} d D_{-}$fn function can be vectorized in the same way to aggregate statistics from an ensemble over different values of the diameter.

This gradient is plotted in Fig. 3 (c). We see that the gradient is positive and constant for $D<0.8$ corresponding to the linear increase in the average energy. Moreover, we see that the derivative crosses zero exactly at the maximum average energy. Finally, we observe that the gradient goes back to zero at $D=1$. This suggests that $D=0.8$ is the point of maximum disorder, as we found by brute force above. It also shows that $D=1$ is the minimum energy configuration of the diameter. Although we hadn't hypothesized it, we realize this must be true since $D<1$ states are symmetric with $D>1$ as we keep the total packing fraction constant.

\subsection{An Energy Model for Flocking ${ }^{4}$}

We will now use the primitives provided by JAX MD to build a flocking simulation inspired by Reynolds' landmark paper [79]. This is a simulation that looks very dissimilar from most conventional physics simulations. Nonetheless, the primitives that JAX MD provides allow us to write the simulation in such a way that it scales it to tens-of-thousands of agents (that Reynolds calls "boids"). Reynolds notes that in nature flocks do not seem to have a maximum size, but instead can keep acquiring new boids and grow without bound. He also comments that each boid cannot possibly be keeping track of the entire flock and must, instead, be focusing on its local neighborhood. As such, we will use neighbor lists to keep track of nearby boids and scale the simulation.

Reynolds proposes three simple and local rules that boids might try to follow: alignment causing boids to point in the same direction as their neighbors, avoidance stops boids from colliding with nearby boids, and cohesion that causes boids to point towards the center of mass of their neighbors. We will represent boids by a position, $\vec{r} \in \mathbb{R}^{2}$, and an angle, $\theta$. We will also include disk-like obstacles along with predators. The obstacles will repel both the boids and the predators. The predators will attempt to move themselves towards nearby boids and the boids will flee from the predators. We also allow the predators to "sprint" every-so-often to chase the boids.

Unlike standard boid simulations, we will treat this as an energy model such that low energy configurations satisfy Reynolds' three properties as well as our own constraints on obstacles and predators. As such, we define two energy functions,

$$
\begin{aligned}
E_{\text {boid }} & =E_{\text {align }}+E_{\text {avoid }}+E_{\text {cohesion }}+E_{\text {obstacle }}+E_{\text {boid-predator }} \\
E_{\text {predator }} & =E_{\text {predator-boid }}+E_{\text {obstacle }}
\end{aligned}
$$

\footnotetext{
${ }^{4}$ Colaboratory notebook at: github.com/google/jax-md/notebooks/flocking.ipynb
} 


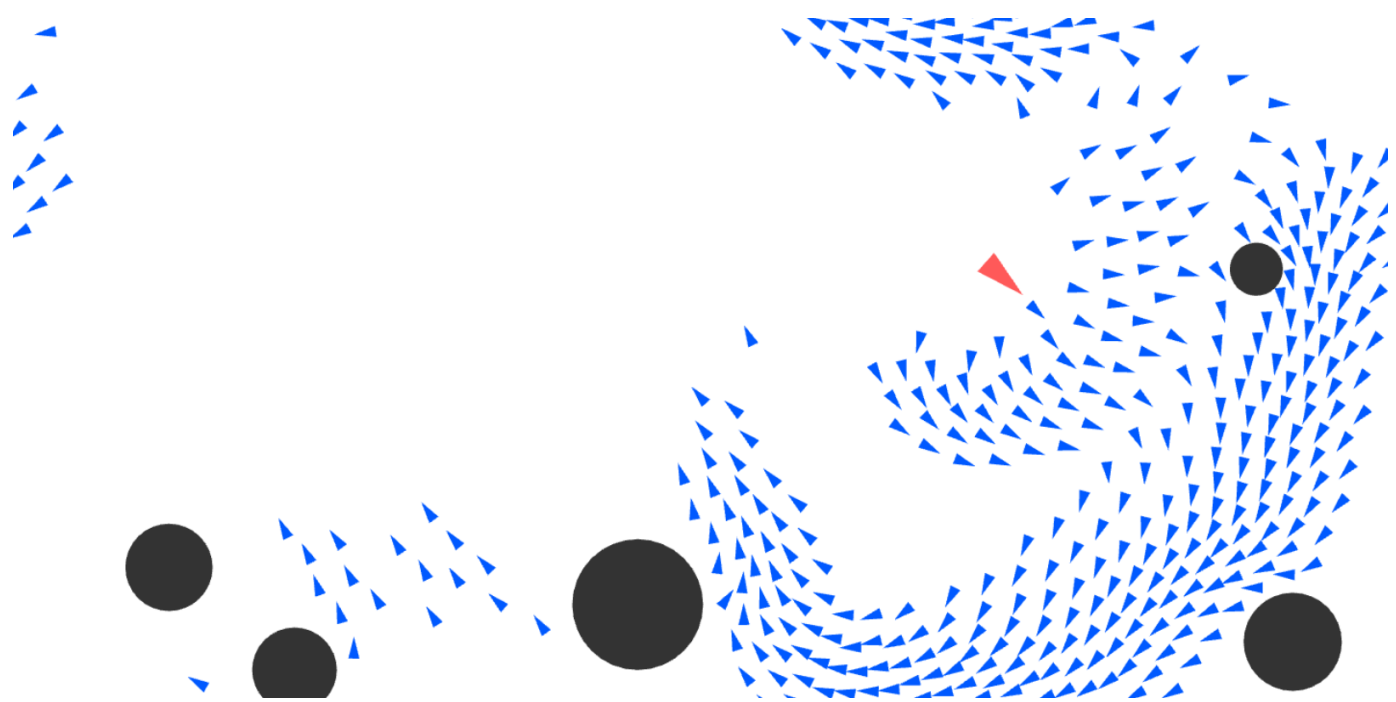

Figure 4: Flocking simulation. Blue arrows represent boids, red arrow represents a predator, and black circles are obstacles.

The simulation will be defined so that boids and predators move in the direction they are pointing at each step, but have their position and orientation updated to minimize their contribution to the above energy. For a detailed discussion of each term in this energy function, see section B.

Although this simulation is only loosely related to standard physics simulations, we still make use of space.periodic to compute displacement and move the boids. We also use neighbor lists so that each boid only has to consider its local neighborhood. A figure showing the behavior of the simulation can be seen in fig. 4. The boids move cohesively and swarm around obstacles while the predators chase them around the simulation. While this simulation can now be used as a standard flocking simulation, JAX MD allows us to take it a step further. Since the simulation is differentiable with respect to the parameters that control the agents, we can use gradients to train boids and predators as a two-player game (potentially controlled by neural networks), ensemble populations for efficient evolution, and meta-learn environmental hyperparameters for interesting emergent behavior. This would be an interesting avenue for future experiments.

\section{Conclusion}

We have described recent work on a general purpose differentiable physics package. We have demonstrated several instances where JAX MD can simplify research and accelerate researcher iteration time. We believe that JAX MD will enable new avenues of research combining developments from machine learning with the physical sciences.

\section{Broader Impact}

We believe there is significant potential when deep learning, automatic differentiation, and simulation meet. We are excited about the possibilities of larger and more accurate simulations of quantum mechanical systems. If we can reliably simulate and optimize these systems at scale it has the potential to revolutionize many problems that are currently hard: from material design to drug discovery. Of course, if this program is successful enough to provide actual benefits to benevolent technologies it surely has the potential to be used in an ethically questionable manner to e.g. design weapons. We are also excited about the potential of marrying the extreme expressivity of machine learning models with the excellent generalization capabilities of physical laws to improve our ability to do science. 


\section{Acknowledgments and Disclosure of Funding}

We are indebted to early users of JAX MD who provided valuable feedback on early versions of the project, especially Carl Goodrich, Ella King, Michael Brenner, Kareem Hegazy, Ryan Coffee, Rouxia Chen, Matheiu Bauchy, Luke Metz, and Amil Merchant. We would especially like to thank Carl Goodrich for contributions to the library, including the custom potential colaboratory notebook linked in section 3.2.2. We would also like to thank Rouxia Chen for rigorously testing and improving the BKS potential. We thank the entire JAX and XLA teams for making making such outstanding libraries that we were able to build on. In particular, we appreciate useful feedback and assistance from Matthew Johnson, Skye Wanderman-Milne, James Bradbury, and Roy Frostig. In addition to JAX, we learned a great deal and were inspired by excellent classical MD packages such as LAMMPS, HOOMD Blue, and OpenMM, as well as the Graph Network library by DeepMind and, in particular, we thank Thomas Keck for useful advice. We are additionally grateful Jascha Sohl-Dickstein and Yutong Zhao for useful discussions along with the Google Brain team for their help and support. Google is the sole source of funding for this work.

\section{References}

[1] Giuseppe Carleo, Ignacio Cirac, Kyle Cranmer, Laurent Daudet, Maria Schuld, Naftali Tishby, Leslie Vogt-Maranto, and Lenka Zdeborová. Machine learning and the physical sciences. Reviews of Modern Physics, 91(4):045002, 2019.

[2] Kyle Cranmer, Johann Brehmer, and Gilles Louppe. The frontier of simulation-based inference. Proceedings of the National Academy of Sciences, 2020.

[3] Grégoire Montavon, Katja Hansen, Siamac Fazli, Matthias Rupp, Franziska Biegler, Andreas Ziehe, Alexandre Tkatchenko, Anatole V Lilienfeld, and Klaus-Robert Müller. Learning invariant representations of molecules for atomization energy prediction. In Advances in neural information processing systems, pages 440-448, 2012.

[4] Justin Gilmer, Samuel S Schoenholz, Patrick F Riley, Oriol Vinyals, and George E Dahl. Neural message passing for quantum chemistry. In Proceedings of the 34th International Conference on Machine Learning-Volume 70, pages 1263-1272. JMLR. org, 2017.

[5] Kristof Schütt, Pieter-Jan Kindermans, Huziel Enoc Sauceda Felix, Stefan Chmiela, Alexandre Tkatchenko, and Klaus-Robert Müller. Schnet: A continuous-filter convolutional neural network for modeling quantum interactions. In Advances in neural information processing systems, pages 991-1001, 2017.

[6] Kristof T Schütt, Farhad Arbabzadah, Stefan Chmiela, Klaus R Müller, and Alexandre Tkatchenko. Quantum-chemical insights from deep tensor neural networks. Nature communications, 8(1):1-8, 2017.

[7] Albert P Bartók, Mike C Payne, Risi Kondor, and Gábor Csányi. Gaussian approximation potentials: The accuracy of quantum mechanics, without the electrons. Physical review letters, 104(13):136403, 2010.

[8] Albert P Bartók, James Kermode, Noam Bernstein, and Gábor Csányi. Machine learning a general-purpose interatomic potential for silicon. Physical Review X, 8(4):041048, 2018.

[9] Nongnuch Artrith, Alexander Urban, and Gerbrand Ceder. Constructing first-principles phase diagrams of amorphous li x si using machine-learning-assisted sampling with an evolutionary algorithm. The Journal of chemical physics, 148(24):241711, 2018.

[10] Nongnuch Artrith, Tobias Morawietz, and Jörg Behler. High-dimensional neural-network potentials for multicomponent systems: Applications to zinc oxide. Physical Review B, 83(15): 153101, 2011.

[11] Atsuto Seko, Akira Takahashi, and Isao Tanaka. Sparse representation for a potential energy surface. Physical Review B, 90(2):024101, 2014.

[12] Miles Cranmer, Sam Greydanus, Stephan Hoyer, Peter Battaglia, David Spergel, and Shirley Ho. Lagrangian neural networks. arXiv preprint arXiv:2003.04630, 2020. 
[13] Alvaro Sanchez-Gonzalez, Jonathan Godwin, Tobias Pfaff, Rex Ying, Jure Leskovec, and Peter W Battaglia. Learning to simulate complex physics with graph networks. arXiv preprint arXiv:2002.09405, 2020.

[14] Alvaro Sanchez-Gonzalez, Victor Bapst, Kyle Cranmer, and Peter Battaglia. Hamiltonian graph networks with ode integrators. arXiv preprint arXiv:1909.12790, 2019.

[15] Miles D Cranmer, Rui Xu, Peter Battaglia, and Shirley Ho. Learning symbolic physics with graph networks. arXiv preprint arXiv:1909.05862, 2019.

[16] Samuel S Schoenholz, Ekin D Cubuk, Daniel M Sussman, Efthimios Kaxiras, and Andrea J Liu. A structural approach to relaxation in glassy liquids. Nature Physics, 12(5):469, 2016.

[17] Samuel S. Schoenholz, Ekin D. Cubuk, Efthimios Kaxiras, and Andrea J. Liu. Relationship between local structure and relaxation in out-of-equilibrium glassy systems. Proceedings of the National Academy of Sciences, 114(2):263-267, 2017. ISSN 0027-8424. doi: 10.1073/pnas. 1610204114. URL https://www. pnas .org/content/114/2/263.

[18] E. D. Cubuk, R. J. S. Ivancic, S. S. Schoenholz, D. J. Strickland, et al. Structure-property relationships from universal signatures of plasticity in disordered solids. Science, 358(6366): 1033-1037, 2017. ISSN 0036-8075. doi: 10.1126/science.aai8830. URL https : //science. sciencemag.org/content/358/6366/1033.

[19] Victor Bapst, Thomas Keck, A Grabska-Barwińska, Craig Donner, Ekin Dogus Cubuk, SS Schoenholz, Annette Obika, AWR Nelson, Trevor Back, and Demis Hassabis. Unveiling the predictive power of static structure in glassy systems. Nature Physics, 16(4):448-454, 2020.

[20] Andrew W Senior, Richard Evans, John Jumper, James Kirkpatrick, Laurent Sifre, Tim Green, Chongli Qin, Augustin Žídek, Alexander WR Nelson, Alex Bridgland, et al. Improved protein structure prediction using potentials from deep learning. Nature, pages 1-5, 2020.

[21] John Jumper et al. High accuracy protein structure prediction using deep learning. In Fourteenth Critical Assessment of Techniques for Protein Structure Prediction, pages 22-25, 2020.

[22] John Ingraham, Adam Riesselman, Chris Sander, and Debora Marks. Learning protein structure with a differentiable simulator. 2018.

[23] Mohammed AlQuraishi. End-to-end differentiable learning of protein structure. Cell systems, 8 (4):292-301, 2019.

[24] Momchil Minkov, Ian AD Williamson, Lucio C Andreani, Dario Gerace, Beicheng Lou, Alex Y Song, Tyler W Hughes, and Shanhui Fan. Inverse design of photonic crystals through automatic differentiation. arXiv preprint arXiv:2003.00379, 2020.

[25] Stephan Hoyer, Jascha Sohl-Dickstein, and Sam Greydanus. Neural reparameterization improves structural optimization. arXiv preprint arXiv:1909.04240, 2019.

[26] Brenden M Lake, Ruslan Salakhutdinov, and Joshua B Tenenbaum. Human-level concept learning through probabilistic program induction. Science, 350(6266):1332-1338, 2015.

[27] Steve Plimpton. Fast parallel algorithms for short-range molecular dynamics. Journal of computational physics, 117(1):1-19, 1995.

[28] Joshua A Anderson, Chris D Lorenz, and Alex Travesset. General purpose molecular dynamics simulations fully implemented on graphics processing units. Journal of computational physics, 227(10):5342-5359, 2008.

[29] Jens Glaser, Trung Dac Nguyen, Joshua A Anderson, Pak Lui, Filippo Spiga, Jaime A Millan, David C Morse, and Sharon C Glotzer. Strong scaling of general-purpose molecular dynamics simulations on gpus. Computer Physics Communications, 192:97-107, 2015. 
[30] Peter Eastman, Jason Swails, John D Chodera, Robert T McGibbon, Yutong Zhao, Kyle A Beauchamp, Lee-Ping Wang, Andrew C Simmonett, Matthew P Harrigan, Chaya D Stern, et al. Openmm 7: Rapid development of high performance algorithms for molecular dynamics. PLoS computational biology, 13(7):e1005659, 2017.

[31] Albert P Bartók and Gábor Csányi. G aussian approximation potentials: A brief tutorial introduction. International Journal of Quantum Chemistry, 115(16):1051-1057, 2015.

[32] Nongnuch Artrith and Alexander Urban. An implementation of artificial neural-network potentials for atomistic materials simulations: Performance for tio2. Computational Materials Science, 114:135-150, 2016.

[33] Nongnuch Artrith, Alexander Urban, and Gerbrand Ceder. Efficient and accurate machinelearning interpolation of atomic energies in compositions with many species. Physical Review B, 96(1):014112, 2017.

[34] Berk Onat, Ekin D Cubuk, Brad D Malone, and Efthimios Kaxiras. Implanted neural network potentials: Application to li-si alloys. Physical Review B, 97(9):094106, 2018.

[35] Rainier Barrett, Maghesree Chakraborty, Dilnoza B Amirkulova, Heta A Gandhi, Geemi P Wellawatte, and Andrew D White. Hoomd-tf: Gpu-accelerated, online machine learning in the hoomd-blue molecular dynamics engine. Journal of Open Source Software, 5(51):2367, 2020.

[36] April M Cooper, Johannes Kästner, Alexander Urban, and Nongnuch Artrith. Efficient training of ann potentials by including atomic forces via taylor expansion and application to water and a transition-metal oxide. npj Computational Materials, 6(1):1-14, 2020.

[37] James Bradbury, Roy Frostig, Peter Hawkins, Matthew James Johnson, Chris Leary, Dougal Maclaurin, and Skye Wanderman-Milne. JAX: composable transformations of Python+NumPy programs, 2018. URL http://github.com/google/jax.

[38] Roy Frostig, Matthew James Johnson, and Chris Leary. Compiling machine learning programs via high-level tracing, 2018.

[39] Fernando Pérez and Brian E. Granger. IPython: a system for interactive scientific computing. Computing in Science and Engineering, 9(3):21-29, May 2007. ISSN 1521-9615. doi: 10.1109/ MCSE.2007.53. URL https://ipython.org.

[40] Atilim Gunes Baydin, Barak A Pearlmutter, Alexey Andreyevich Radul, and Jeffrey Mark Siskind. Automatic differentiation in machine learning: a survey. Journal of machine learning research, 18(153), 2018.

[41] Teresa Tamayo-Mendoza, Christoph Kreisbeck, Roland Lindh, and Alán Aspuru-Guzik. Automatic differentiation in quantum chemistry with applications to fully variational hartree-fock. ACS central science, 4(5):559-566, 2018.

[42] J-D Müller and P Cusdin. On the performance of discrete adjoint cfd codes using automatic differentiation. International journal for numerical methods in fluids, 47(8-9):939-945, 2005.

[43] Jeffrey P Thomas, Earl H Dowell, and Kenneth C Hall. Using automatic differentiation to create a nonlinear reduced-order-model aerodynamic solver. AIAA journal, 48(1):19-24, 2010.

[44] Christian H Bischof, H Martin Bücker, Arno Rasch, Emil Slusanschi, and Bruno Lang. Automatic differentiation of the general-purpose computational fluid dynamics package fluent. Journal of fluids engineering, 129(5):652-658, 2007.

[45] Luca Capriotti. Fast greeks by algorithmic differentiation. Available at SSRN 1619626, 2010.

[46] Isabelle Charpentier and Mohammed Ghemires. Efficient adjoint derivatives: application to the meteorological model meso-nh. Optimization Methods and Software, 13(1):35-63, 2000.

[47] Gregory R Carmichael, Adrian Sandu, et al. Sensitivity analysis for atmospheric chemistry models via automatic differentiation. Atmospheric Environment, 31(3):475-489, 1997. 
[48] Andrea Walther. Automatic differentiation of explicit runge-kutta methods for optimal control. Computational Optimization and Applications, 36(1):83-108, 2007.

[49] Filipe de Avila Belbute-Peres, Kevin Smith, Kelsey Allen, Josh Tenenbaum, and J Zico Kolter. End-to-end differentiable physics for learning and control. In Advances in Neural Information Processing Systems, pages 7178-7189, 2018.

[50] Maria Schuld, Ville Bergholm, Christian Gogolin, Josh Izaac, and Nathan Killoran. Evaluating analytic gradients on quantum hardware. Physical Review A, 99(3):032331, 2019.

[51] Bharath Ramsundar, Peter Eastman, Patrick Walters, Vijay Pande, Karl Leswing, and Zhenqin Wu. Deep Learning for the Life Sciences. O'Reilly Media, 2019. https : / www . amazon . com/ Deep-Learning-Life-Sciences-Microscopy/dp/1492039837.

[52] Martín Abadi, Paul Barham, Jianmin Chen, Zhifeng Chen, Andy Davis, Jeffrey Dean, Matthieu Devin, Sanjay Ghemawat, Geoffrey Irving, Michael Isard, et al. Tensorflow: A system for large-scale machine learning. In 12th \{USENIX\} Symposium on Operating Systems Design and Implementation ( $\{O S D I\} 16)$, pages 265-283, 2016.

[53] Adam Paszke, Sam Gross, Soumith Chintala, Gregory Chanan, Edward Yang, Zachary DeVito, Zeming Lin, Alban Desmaison, Luca Antiga, and Adam Lerer. Automatic differentiation in pytorch. 2017.

[54] Ronan Collobert, Samy Bengio, and Johnny Mariéthoz. Torch: a modular machine learning software library. Technical report, Idiap, 2002.

[55] Frédéric Bastien, Pascal Lamblin, Razvan Pascanu, James Bergstra, Ian Goodfellow, Arnaud Bergeron, Nicolas Bouchard, David Warde-Farley, and Yoshua Bengio. Theano: new features and speed improvements. arXiv preprint arXiv:1211.5590, 2012.

[56] Dougal Maclaurin, David Duvenaud, and Ryan P Adams. Autograd: Effortless gradients in numpy. In ICML 2015 AutoML Workshop, volume 238, 2015.

[57] Mike Innes, Alan Edelman, Keno Fischer, Chris Rackauckus, Elliot Saba, Viral B Shah, and Will Tebbutt. Zygote: A differentiable programming system to bridge machine learning and scientific computing. arXiv preprint arXiv:1907.07587, 2019.

[58] Yuanming Hu, Luke Anderson, Tzu-Mao Li, Qi Sun, Nathan Carr, Jonathan Ragan-Kelley, and Frédo Durand. Difftaichi: Differentiable programming for physical simulation. arXiv preprint arXiv:1910.00935, 2019.

[59] Yuanming Hu, Tzu-Mao Li, Luke Anderson, Jonathan Ragan-Kelley, and Frédo Durand. Taichi: a language for high-performance computation on spatially sparse data structures. ACM Transactions on Graphics (TOG), 38(6):1-16, 2019.

[60] Stefan Van Der Walt, S Chris Colbert, and Gael Varoquaux. The numpy array: a structure for efficient numerical computation. Computing in Science \& Engineering, 13(2):22, 2011.

[61] Tom Hennigan, Trevor Cai, Tamara Norman, and Igor Babuschkin. Haiku: Sonnet for JAX, 2020. URL http://github.com/deepmind/dm-haiku.

[62] Jörg Behler. Atom-centered symmetry functions for constructing high-dimensional neural network potentials. The Journal of chemical physics, 134(7):074106, 2011.

[63] Ekin D Cubuk, Samuel Stern Schoenholz, Jennifer M Rieser, Brad Dean Malone, Joerg Rottler, Douglas J Durian, Efthimios Kaxiras, and Andrea J Liu. Identifying structural flow defects in disordered solids using machine-learning methods. Physical review letters, 114(10):108001, 2015.

[64] Ekin D Cubuk, Andrea J Liu, Efthimios Kaxiras, and Samuel S Schoenholz. Unifying framework for strong and fragile liquids via machine learning: a study of liquid silica. arXiv preprint arXiv:2008.09681, 2020.

[65] Jörg Behler and Michele Parrinello. Generalized neural-network representation of highdimensional potential-energy surfaces. Physical review letters, 98(14):146401, 2007. 
[66] Peter W Battaglia, Jessica B Hamrick, Victor Bapst, Alvaro Sanchez-Gonzalez, Vinicius Zambaldi, Mateusz Malinowski, Andrea Tacchetti, David Raposo, Adam Santoro, Ryan Faulkner, et al. Relational inductive biases, deep learning, and graph networks. arXiv preprint arXiv:1806.01261, 2018.

[67] Glenn J Martyna, Michael L Klein, and Mark Tuckerman. Nosé-hoover chains: The canonical ensemble via continuous dynamics. The Journal of chemical physics, 97(4):2635-2643, 1992.

[68] Erik Bitzek, Pekka Koskinen, Franz Gähler, Michael Moseler, and Peter Gumbsch. Structural relaxation made simple. Physical review letters, 97(17):170201, 2006.

[69] Walter Kob and Hans C Andersen. Testing mode-coupling theory for a supercooled binary lennard-jones mixture i: The van hove correlation function. Physical Review E, 51(5):4626, 1995.

[70] Corey S O'hern, Leonardo E Silbert, Andrea J Liu, and Sidney R Nagel. Jamming at zero temperature and zero applied stress: The epitome of disorder. Physical Review E, 68(1):011306, 2003.

[71] BWH Van Beest, Gert Jan Kramer, and RA Van Santen. Force fields for silicas and aluminophosphates based on ab initio calculations. Physical Review Letters, 64(16):1955, 1990.

[72] Antoine Carré, Simona Ispas, Jürgen Horbach, and Walter Kob. Developing empirical potentials from ab initio simulations: The case of amorphous silica. Computational Materials Science, 124:323-334, 2016.

[73] Han Liu, Zipeng Fu, Yipeng Li, Nazreen Farina Ahmad Sabri, and Mathieu Bauchy. Machine learning forcefield for silicate glasses. arXiv preprint arXiv:1902.03486, 2019.

[74] Raju P Gupta. Lattice relaxation at a metal surface. Physical Review B, 23(12):6265, 1981.

[75] Philip M Morse. Diatomic molecules according to the wave mechanics. ii. vibrational levels. Physical Review, 34(1):57, 1929.

[76] Frank H. Stillinger and Thomas A. Weber. Computer simulation of local order in condensed phases of silicon. Phys. Rev. B, 31:5262-5271, Apr 1985. doi: 10.1103/PhysRevB.31.5262. URL https://link.aps.org/doi/10.1103/PhysRevB.31.5262.

[77] Murray S Daw and Michael I Baskes. Embedded-atom method: Derivation and application to impurities, surfaces, and other defects in metals. Physical Review B, 29(12):6443, 1984.

[78] Ekin D Cubuk, Brad D Malone, Berk Onat, Amos Waterland, and Efthimios Kaxiras. Representations in neural network based empirical potentials. The Journal of chemical physics, 147(2): 024104, 2017.

[79] Craig W Reynolds. Flocks, herds and schools: A distributed behavioral model. In Proceedings of the 14th annual conference on Computer graphics and interactive techniques, pages 25-34, 1987.

\section{A Differentiable Minimization Routine}

Code for the minimization routine in section 4.2:

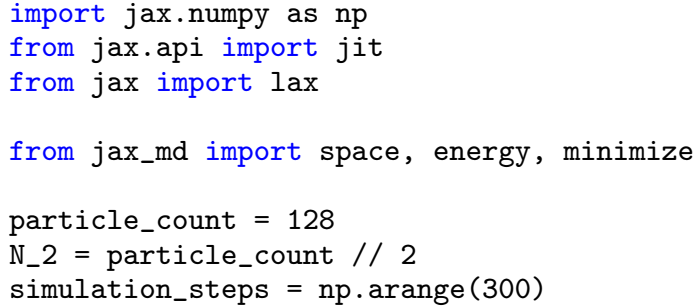




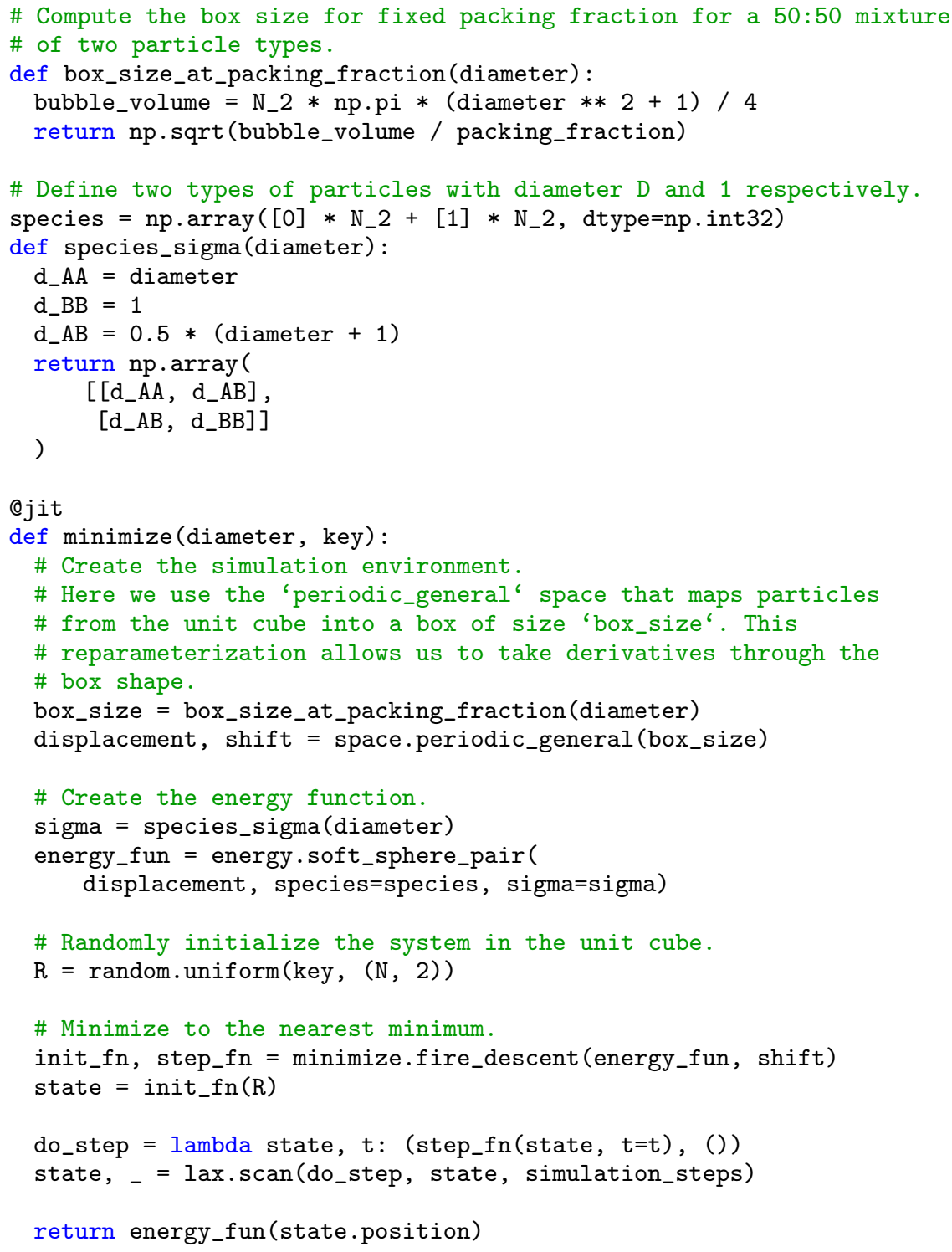

\section{B Flocking Details}

We will now describe details of our attempt to recreate Reynolds' seminal work, "Flocks, Herds, and Schools: A Distributed Behavioral Model" [79] as an energy model. To simplify our discussion, we will focus on a two-dimensional version of Reynolds' simulation.

In nature there are many examples in which large numbers of animals exhibit complex collective motion (schools of fish, flocks of birds, herds of horses, colonies of ants). Reynolds introduces a model of such collective behavior (henceforth refered to as "flocking") based on simple rules that can be computed locally for each entity (referred to as a "boid") in the flock based on its environment. This paper is written in the context of computer graphics and so Reynolds is going for biologically inspired simulations that look right rather than accuracy in any statistical sense. Ultimately, Reynolds measures success in terms of "delight" people find in watching the simulations; we will use a similar metric here. 


\section{B.1 Boids}

Reynolds is interested in simulating bird-like entities that are described by a position, $R \in \mathbb{R}^{2}$, and an orientation, $\theta \in \mathbb{R}$. This state can optionally augmented with extra information (for example, hunger or fear). We can define a Boids type that stores data for a collection of boids as two arrays. $\mathrm{R}$ is an array of shape [boid_count, spatial_dimension] and theta is an array of shape [boid_count]. An individual boid is an index into these arrays. It will often be useful to refer to the vector orientation of the boid $\hat{N}(\theta)=(\cos \theta, \sin \theta)$.

\section{B.2 Dynamics}

Now that we have defined our boids, we have to imbue them with some rules governing their motion. Reynolds notes that in nature flocks do not seem to have a maximum size, but instead can keep acquiring new boids and grow without bound. He also comments that each boid cannot possibly be keeping track of the entire flock and must, instead, be focusing on its local neighborhood. Reynolds then proposes three simple, local, rules that boids might try to follow:

1. Alignment: Boids will try to align themselves in the direction of their neighbors.

2. Avoidance: Boids will avoid colliding with their neighbors.

3. Cohesion: Boids will try to move towards the center of mass of their neighbors.

In his exposition, Reynolds is vague about the details for each of these rules and so we will take some creative liberties. We will try to phrase this problem as an energy model, so our goal will be to write down an "energy" function (similar to a "loss") $E(R, \theta)$ such that low-energy configurations of boids satisfy each of the three rules above.

We will write the total energy as a sum of three terms, one for each of the rules above:

$$
E(R, \theta)=E_{\text {Align }}(R, \theta)+E_{\text {Avoid }}(R, \theta)+E_{\text {Cohesion }}(R, \theta)
$$

We will discuss each of these rules separately below starting with alignment. Of course, any of these terms could be replaced by a learned solution.

Once we have an energy defined in this way, configurations of boids that move along low energy trajectories might display behavior that looks appealing. However, we still have a lot of freedom to decide how we want to define dynamics over the boids. Reynolds says he uses overdamped dynamics and so we will do something similar. In particular, we will update the position of the boids so that they try to move to minimize their energy. Simultaneously, we assume that the boids are swimming (or flying / walking). We choose a particularly simple model of this to start with and assume that the boids move at a fixed speed, $v$, along whatever direction they are pointing. We will use simple forward-Euler integration. This gives an update step,

$$
R_{i}^{\prime}=R_{i}+\delta t\left(v \hat{N}\left(\theta_{i}\right)-\nabla_{R_{i}} E(R, \theta)\right)
$$

where $\delta t$ is a timestep that we are allowed to choose.

We will update the orientations of the boids turn them towards "low energy" directions. To do this we will once again use a simple forward-Euler scheme,

$$
\theta_{i}^{\prime}=\theta_{i}-\delta t \nabla_{\theta_{i}} E(R, \theta) .
$$

This is one choice of dynamics, however many choices would produce aesthetically pleasing motion.

\section{B.2.1 Locality}

We will now describe the three contributions to the total energy beginning with alignment. However, it is useful to setup some notation and discuss the idea of locality, which will be common to all three terms in the energy. When writing down these rules, it is often easier to express them for a single pair of boids and then use automatic vectorization via vmap to extend them to the entire simulation. To that end, we consider a pair of boids, denoted $i$ and $j$ respectively. We define $\vec{R}_{i j}=\vec{R}_{i}-\vec{R}_{j}$ be the displacement vector from boid $j$ to boid $i$. 
As discussed above, a key assumption that Reynolds makes is that each individual boid only interacts with nearby boids. To ensure that the different terms in eqn. (3) are local, we will use the function,

$$
\rho\left(R_{i j} ; R_{c}\right)= \begin{cases}\frac{1}{\alpha}\left(1-\frac{\left\|R_{i j}\right\|}{R_{c}}\right)^{\alpha} & \left\|R_{i j}\right\|<R_{c} \\ 0 & \text { otherwise }\end{cases}
$$

to ensure that the energies go to zero continuously when boids are greater than a distance $R_{c}$ apart.

\section{B.2.2 Alignment}

The goal of the alignment term is to coerce boids to point in the same direction. As such, we choose a simple proposal with two parameters:

$$
\epsilon_{\text {Align }}\left(R_{i j}, \theta_{i}, \theta_{j}\right)=J_{\text {Align }} \rho\left(R_{i j}, R_{\text {Align }}\right)\left(1-\hat{N}\left(\theta_{i}\right) \cdot \hat{N}\left(\theta_{j}\right)\right)^{2} .
$$

Here $J_{\text {Align }}$ determines the strength of the alignment term and $R_{\text {Align }}$ dictates its range. This energy will be maximized when $N\left(\theta_{i}\right)$ and $N\left(\theta_{j}\right)$ are anti-aligned and minimized when $\hat{N}\left(\theta_{i}\right)=\hat{N}\left(\theta_{j}\right)$. In general, we would like our boids to turn to align themselves with their neighbors rather than shift their centers to move apart. Therefore, we'll insert a stop-gradient into the displacement.

\section{B.2.3 Avoidance}

We can incorporate an avoidance rule that will keep the boids from bumping into one another. This will help them to form a flock with some volume rather than collapsing together. To this end, imagine a very simple model of boids that push away from one another if they get within a distance $R_{\text {Avoid }}$. To do this we simply use the cutoff function described in eqn. (6) along with a scale $J_{\text {Avoid }}$ that gives the magnitude of the avoidance term.

$$
\epsilon_{\text {Avoid }}\left(R_{i j}\right)=J_{\text {Avoid }} \rho\left(R_{i j}, R_{\text {Avoid }}\right) .
$$

\section{B.2.4 Cohesion}

The final piece of Reynolds' boid model is cohesion. In simulations without a cohesion term, the boids tend to move in the same direction but they also often drift apart. To make the boids behave more like schools of fish or birds, which maintain a more compact arrangement, we add a cohesion term to the energy.

The goal of the cohesion term is to align boids towards the center of mass of their neighbors. Given a boid, $i$, we can compute the center of mass position of its neighbors as,

$$
\Delta R_{i}=\frac{1}{|\mathcal{N}|} \sum_{j \in \mathcal{N}} R_{i j}
$$

where we have let $\mathcal{N}$ be the set of boids such that $\left\|R_{i j}\right\|<R_{\text {Cohesion, }}$, the range of the cohesion term. Given the center of mass displacements, we can define a reasonable cohesion energy as,

$$
\epsilon_{\text {Cohesion }}\left(\widehat{\Delta R}_{i}, N_{i}\right)=\frac{1}{2} J_{\text {Cohesion }}\left(1-\widehat{\Delta R}_{i} \cdot N\right)^{2}
$$

where $\widehat{\Delta R}_{i}=\Delta R_{i} /\left\|\Delta R_{i}\right\|$ is the normalized vector pointing in the direction of the center of mass. This function is minimized when the boid is pointing in the direction of the center of mass. This term can be slightly improved by only taking into account boids in the line of sight of the central boid.

\section{B.3 Extras}

This describes the core elements of Reynolds' simulation. However, there are several additional elements that can easily be added to the energy model to make the visualization more interesting.

\section{B.3.1 Obstacles}

The first thing we'll add are obstacles that the boids and the predators will try to avoid as they wander around the simulation. For the purposes of this notebook, we'll restrict ourselves to disk-like obstacles. Each disk will be described by a center position and a radius, $D_{\text {Obstacle }}$. 
In a similar spirit to the energy functions above, we would like a function that encourages the boids to avoid obstacles. To that end, for an obstacle $o$ and boid $i$ we use the following functional form,

$$
\epsilon_{\text {Obstacle }}\left(R_{i o}, N_{i}, D_{o}\right)=J_{\text {Obstacle }} \rho\left(R_{i o}, R_{\text {Obstacle }}\right)\left(1+N_{i} \cdot \widehat{R_{i o}}\right)^{2}
$$

for $R_{i o}$ the displacement vector between a boid $i$ and an obstacle $o$. This energy is zero when the boid and the obstacle are not overlapping. When they are overlapping, the energy is minimized when the boid is facing away from the obstacle.

\section{B.3.2 Predators}

Next we are going to introduce some predators into the environment for the boids to run away from. Much like the boids, the predators will be described by a position and an angle.

The predators will also follow similar dynamics to the boids, swimming in whatever direction they are pointing at some speed that we can choose. Unlike in the previous versions of the simulation, predators naturally introduce some asymmetry to the system. In particular, we would like the boids to flee from the predators, but we want the predators to chase the boids. To achieve this behavior, we will consider a system reminiscient of a two-player game in which the boids move to minimize an energy,

$$
E_{\text {Boid }}=E_{\text {Align }}+E_{\text {Avoid }}+E_{\text {Cohesion }}+E_{\text {Obstacle }}+E_{\text {Boid-Predator }} .
$$

Simultaneously, the predators move in an attempt to minimize a simpler energy,

$$
E_{\text {Predator }}=E_{\text {Predator-Boid }}+E_{\text {Obstacle }} \text {. }
$$

To add predators to the environment we therefore need to add two rules, one that dictates the boids behavior near a predator and one for the behavior of predators near a group of boids. In both cases we will see that we can draw significant inspiration from behaviors that we've already developed. In particular, for the boid-predator function, we will use the same obstacle avoiding energy above. In this way, the boids will flee if a predator gets too close. Finally, for the predator-boid function, we will reuse the cohesion function above. Thus, predators will move towards the center of mass of groups of nearby boids. 